\title{
Robust Autopilot Design for Landing a Large Civil Aircraft in Crosswind ${ }^{\text {th }}$
}

\author{
Julian Theis $^{\mathrm{a}, *}$, Daniel Ossmann ${ }^{\mathrm{b}}$, Frank Thielecke ${ }^{\mathrm{a}}$, Harald Pfifer ${ }^{\mathrm{c}}$ \\ ${ }^{a}$ Institute of Aircraft Systems Engineering, Hamburg University of Technology, Hamburg, Germany \\ ${ }^{b}$ Institute of System Dynamics and Control, German Aerospace Center (DLR) Oberpfaffenhofen, Wessling, Germany \\ ${ }^{c}$ Mechanical Engineering Department, University of Nottingham, Nottingham, United Kingdom
}

\begin{abstract}
A comprehensive autolanding design for a representative model of a twin-engined commercial aircraft is presented in this paper. To facilitate the design task and minimize control law switching, a cascaded control structure is selected which resembles integrator chains. Classical loopshaping and robust control techniques are used to design the individual control loops. The emphasis is on providing a complete and comprehensive qualitative design strategy. The control system's ability to safely land the aircraft despite strong crosswind in a variety of possible scenarios is demonstrated in an industry-grade verification campaign. Nonlinear Monte Carlo simulations of the airliner model are used to assess the risk of unsafe landing conditions and provide insight into the performance characteristics and limitations of the proposed control system.
\end{abstract}

Keywords: aircraft control, flight control, cascade control, control design, mixed sensitivity problem, H-infinity control

\section{Introduction}

Automatic control systems play a fundamental role in modern civil aviation and are by now capable of assisting the pilot in all flight segments. In fact, today's autopilots can perform challenging maneuvers such as to land the aircraft in poor visibility. To safely land the aircraft, the autopilot must achieve a very high level of precision in a variety of different scenarios. Crosswind poses one of the most severe dangers to landing aircraft. The autolanding system of the A320, e.g., is certified to perform safe landings in crosswind up to 20 knots. For comparison, the demonstrated crosswind in manual flight operation (that requires clear sight of the runway) on the A320 is 35 knots. Improving the ability to handle adverse wind conditions is thus important to increase performance and availability of future autolanding systems. Consequently, several researchers have investigated the potential of modern control techniques for this application, e.g., Holley and Bryson (1977); Shue and Agarwal (1999); Looye et al. (2001); Looye and Joos (2006); de Bruin and Jones (2016). Others have focused on particular subtasks such as the "flare" maneuver immediately before touchdown, e. g., Kaminer and Khargonekar (1990); Biannic and Apkarian (2001); Navarro-Tapia et al. (2017).

The present article details the design of a complete autolanding system for the representative model of a twin-engined commercial transport aircraft in landing configuration. The airliner model was introduced by Biannic and Roos (2015) and

\footnotetext{
This research did not receive any specific grant from funding agencies in the public, commercial, or not-for-profit sectors.

${ }^{*}$ Corresponding author.

Email addresses: julian.theis@tuhh.de (Julian Theis), daniel.ossmann@dlr.de (Daniel Ossmann), frank.thielecke@dlr.de (Frank Thielecke), harald.pfifer@nottingham.ac.uk (Harald Pfifer)
}

is openly available from http://w3. onera.fr/smac/?q= aircraftModel. It was used in a design challenge formulated by Biannic and Boada-Bauxell (2016) from ONERA and Airbus in which the authors also participated (Theis et al., 2017). The autopilot must steer the aircraft through the final approach starting $300 \mathrm{~m}$ above the runway all the way to touchdown. Available data to perform this task is based on current CAT-III instrument landing systems (ILS) and includes measurements of both vertical and horizontal deviation from the glide path. Success is defined as a gentle touchdown close to the runway centerline with wings level and landing gear aligned with the runway. The requirements are quantified by risk dispersions for the risk of short landing, long landing, hard landing, decentered landing, as well as landing with steep bank angle and landing with steep wheel sideslip angle. These dispersions are calculated through extensive Monte Carlo simulations over a wide range of environmental and system parameters.

The autopilot which is developed in the present article satisfies all requirements for 25 knots crosswind. This crosswind corresponds to the absolute maximum certification specification according to airworthiness code EASA CS-25.237. The design uses classical loopshaping and $H_{\infty}$-norm optimal control. The required background information is provided in Section 2 and a problem formulation that facilitates easy tuning through physically relatable design parameters is developed. A cascaded control structure is proposed in Section 3 to resemble integrator chains. This structure is different from the initial control design (Theis et al., 2017) and selected to make loopshaping design for the subsystems particularly easy. Comprehensive descriptions and insights on all subsystem design tasks are provided in Section 4 and 5. Section 4 details the control system designs for the inner control loops using $H_{\infty}$-norm optimal mixed sensitivity loopshaping. Section 5 details the design of the outer con- 
trol loops through classical loopshaping. The complete design is evaluated in Section 6 in nonlinear Monte Carlo simulations, verifying robust performance.

\section{Loopshaping Control Systems Design}

Let a linear time-invariant (LTI) plant model $P$, an LTI disturbance model $P_{d}$, and an LTI compensator $C$ be given and arranged in the standard unity-feedback loop of Figure 1. The output $y(t) \in \mathbb{R}^{n_{y}}$ collects all measurable signals used to calculate the control signal $u(t) \in \mathbb{R}^{n_{u}}$ and includes the effect of a disturbance $d(t) \in \mathbb{R}^{n_{d}}$ acting on the disturbance model. Additionally, the feedback signal is corrupted by measurement noise $n(t) \in \mathbb{R}^{n_{y}}$. In case only $n_{r}<n_{y}$ outputs are to be tracked, $y_{\text {ref }}(t) \in \mathbb{R}^{n_{y}}$ is without loss of generality taken as $y_{\text {ref }}=\left[\begin{array}{c}I_{n_{r}} \\ 0\end{array}\right] \bar{y}_{\text {ref }}$ with $\bar{y}_{\text {ref }}(t) \in \mathbb{R}^{n_{r}}$. The closed-loop transfer function governing this control loop are

$$
\begin{aligned}
& y=\overbrace{(I+P C)^{-1} P C}^{T}\left(y_{\mathrm{ref}}-n\right)+\overbrace{(I+P C)^{-1} P_{d}}^{S P_{d}} d \\
& u=\underbrace{C(I+P C)^{-1}}_{C S}\left(y_{\mathrm{ref}}-n\right)-\underbrace{C(I+P C)^{-1} P_{d}}_{C S P_{d}} d .
\end{aligned}
$$

They are called the sensitivity $S=(I+P C)^{-1}$, control sensitivity $C S$, disturbance sensitivity $S P_{d}$, and complementary sensitivity $T:=I-S=(I+P C)^{-1} P C$ (e.g. Skogestad and Postlethwaite, 2005).

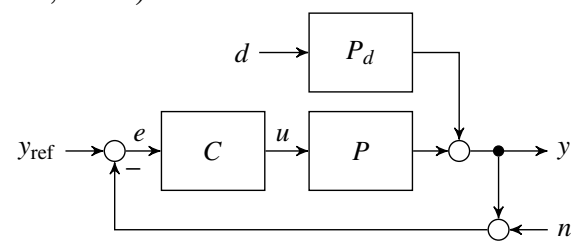

Figure 1: Standard unity feedback loop.

Many properties of feedback control systems can be inferred from the magnitude of these sensitivity functions, e.g., disturbance attenuation levels, tracking capabilities, the frequency range of control activity, and robustness. In general, a control system should reduce the sensitivity $S$ up to a specified frequency to improve disturbance rejection via $S P_{d}$. The relation $T=I-S$ further means that low sensitivity $(S \approx 0)$ over a certain frequency range establishes tracking capabilities for reference signals as $T \approx I$ over this frequency range. The internal model principle (Francis and Wonham, 1975, 1976) can be used to derive desirable sensitivity functions for specific applications. For example, the requirement to follow setpoint changes in all output channels with zero steady-state error can be translated to a sensitivity function

$$
S_{\text {ideal }}=\left[\begin{array}{ccc}
\frac{s}{s+\omega_{1}} & & \\
& \ddots & \\
& & \frac{s}{s+\omega_{n y}}
\end{array}\right],
$$

where $\omega_{i}, i=1, \ldots, n_{y}$ are the desired bandwidths for the individual channels of the multivariable control loop. That is, the ideal sensitivity function has zero steady-state gain, a slope of $+20 \mathrm{~dB}$ per decade in each channel up to the desired bandwidth, and unit gain for higher frequencies. Such an ideal sensitivity is usually impossible to achieve due to Bode's sensitivity integral, as peak magnitude values of greater than 1 are the inevitable result of feedback (cf. Stein, 2003; Skogestad and Postlethwaite, 2005).

To improve transient behavior in response to a reference signal, two-degrees-of-freedom controllers are used. Such controllers consist of feedback $\left(C_{\mathrm{FB}}\right)$ and feedforward $\left(C_{\mathrm{FF}}\right)$ paths, i. e., the control signal is $u=C_{\mathrm{FF}} \bar{y}_{\mathrm{ref}}-C_{\mathrm{FB}} y=K\left[\begin{array}{c}\bar{y}_{\mathrm{ref}} \\ -y\end{array}\right]$. In this case,

$$
\begin{aligned}
y= & \overbrace{\left(I+P C_{\mathrm{FB}}\right)^{-1} P C_{\mathrm{FF}}}^{R} \bar{y}_{\mathrm{ref}} \\
& -\underbrace{\left(I+P C_{\mathrm{FB}}\right)^{-1} P C_{\mathrm{FB}}}_{T} n+\underbrace{\left(I+P C_{\mathrm{FB}}\right)^{-1} P_{d}}_{S P_{d}} d \\
u= & \overbrace{\left(I+C_{\mathrm{FB}} P\right) C_{\mathrm{FF}}}^{S_{\mathrm{i}} C_{\mathrm{FF}}} \bar{y}_{\mathrm{ref}} \\
& -\underbrace{C_{\mathrm{FB}}\left(I+P C_{\mathrm{FB}}\right)^{-1}}_{C_{\mathrm{FB}} S} n-\underbrace{C_{\mathrm{FB}}\left(I+P C_{\mathrm{FB}}\right)^{-1} P_{d}}_{C_{\mathrm{FB}} S P_{d}} d .
\end{aligned}
$$

Hence, reference tracking is governed by a reference transmission function $R$ ( $n_{y}$ outputs and $n_{r}$ inputs) which can be adjusted independently of the sensitivities. The error dynamics with respect to reference signals are described by the map $\left[\begin{array}{c}I_{n_{r}} \\ 0\end{array}\right]-R$, i. e., $e:=y_{\text {ref }}-y=\left(\left[\begin{array}{c}I_{n_{r}} \\ 0\end{array}\right]-R\right) \bar{y}_{\text {ref }}$.

\subsection{Classical Loopshaping}

A classical design technique for single-input-single-output systems is loopshaping [e. g. Horowitz 1963, Doyle et al. 1990]. It is based on "shaping" the loop transfer $L=P C$ such that desirable sensitivity functions are achieved. The ideal sensitivity function (2) translates to an ideal looptransfer $L_{\text {ideal }}=\frac{\omega}{s}$. Hence, the ideal compensator is $C_{\text {ideal }}=\frac{\omega}{s} P^{-1}$. It inverts the plant dynamics and adds integral action. Such a complete inversion is often neither possible nor desirable for reasons of control effort and robustness. Thus, the standard strategy is to select a compensator such the $\frac{\omega}{s}$-loopshape is approximately attained around the desired crossover frequency with sufficient gain in the low-frequency regime. This strategy is well-suited for model-based tuning of simple compensators such as proportional or proportional-integral controllers. It also proves useful for setting the bandwidth of cascaded control systems.

\subsection{Mixed Sensitivity Loopshaping}

Mixed sensitivity loopshaping (e. g. Zhou et al., 1995; Skogestad and Postlethwaite, 2005) seeks to directly shape sensitivity functions through weighted $H_{\infty}$-norm optimization. The requirements are formulated in terms of weighting filters which specify a desired shape, e. g., low sensitivity at low frequencies. The plant model and the weights form a generalized closedloop interconnection, $G$, and a controller can be found from the convex optimization problem

$$
\min _{C_{\mathrm{FB}}, C_{\mathrm{FF}}}\left\|G\left(C_{\mathrm{FB}}, C_{\mathrm{FF}}\right)\right\|_{H_{\infty}} \text {. }
$$


This strategy is particularly useful for multivariable systems, where classical loopshaping is not applicable. Standard numerical tools exist to reliably solve (4), e. g., hinfsyn in Matlab's Robust Control toolbox which implements, among others, the formulation of Glover and Doyle (1988); Doyle et al. (1989). Controller tuning is performed by altering the weights.

The generalized closed-loop interconnection illustrated in Figure 2 is proposed as a weighting scheme which is particularly easy to tune. It corresponds to the input-output map

$$
\begin{gathered}
{\left[\begin{array}{l}
z_{1} \\
z_{2}
\end{array}\right]=\left[\begin{array}{cc}
W_{e} & 0 \\
0 & W_{u}
\end{array}\right]\left[\begin{array}{cc}
D_{e}^{-1} & 0 \\
0 & D_{u}^{-1}
\end{array}\right]} \\
{\left[\begin{array}{ccc}
S & S P_{d} & {\left[\begin{array}{l}
I \\
0
\end{array}\right]-R} \\
C_{\mathrm{FB}} S & C_{\mathrm{FB}} S P_{d} & S_{\mathrm{i}} C_{\mathrm{FF}}
\end{array}\right]\left[\begin{array}{ccc}
D_{e} & 0 & 0 \\
0 & D_{d} & 0 \\
0 & 0 & D_{e}^{r}
\end{array}\right]\left[\begin{array}{l}
w_{1} \\
w_{2} \\
w_{3}
\end{array}\right] .}
\end{gathered}
$$

The outputs $z_{1}, z_{2}$, as well as the inputs $w_{1}, w_{2}, w_{3}$ are fictitious and represent any signal injected or measured at that particular point within the control loop.

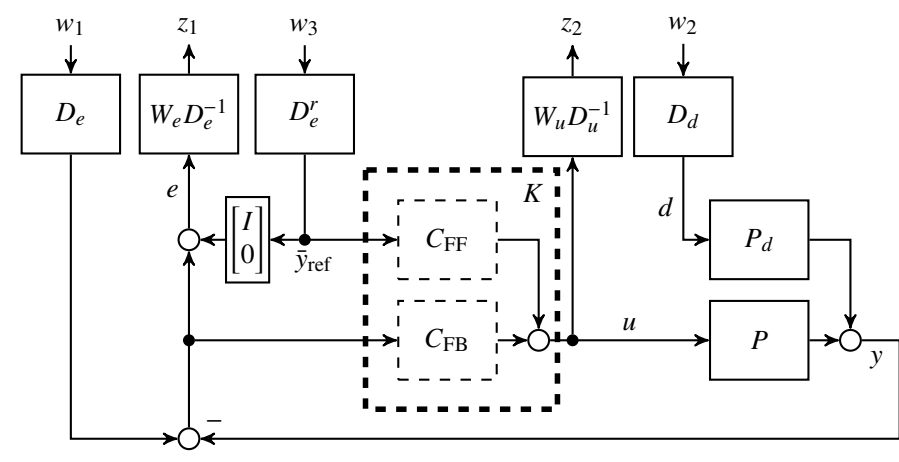

Figure 2: Generalized closed-loop interconnection for two-degrees-of-freedom design.

The sensitivity functions are weighted through two different mechanisms. Shaping filters $W_{e}$ and $W_{u}$, i.e., frequencydependent weights, reflect the general design objectives. Scaling matrices $D_{e}, D_{u}$, and $D_{d}$, i. e., static weights, are used as the main tuning knobs. Using scalings is customary in $H_{\infty}$ control but usually controllers are designed for scaled models $\tilde{P}:=D_{e}^{-1} P D_{u}$ and $\tilde{P}_{d}:=D_{e}^{-1} P_{d} D_{d}$ (cf. Skogestad and Postlethwaite, 2005). Doing so can, however, obscure the fact that scalings inevitably act as additional weights which in return can complicate tuning. Figure 3 shows the completely equivalent formulation using scaled models instead of explicitly representing the scalings as weights. In this case, the actual controller is found to be $K=D_{u} \tilde{K}\left[\begin{array}{cc}D_{e}^{r} & D_{e}\end{array}\right]^{-1}$.

All weights are selected to be diagonal, such that their influence is relatable to physical inputs and outputs. A default choice for the entries of the shaping filter $W_{e}$ are first-order transfer functions with approximately integral behavior up to desired bandwidths $\omega_{\mathrm{b}, i}, i=1, \ldots, n_{r}$ and gain 0.5 beyond that frequency for controlled outputs. The rationale is to decrease (disturbance) sensitivity up to $\omega_{\mathrm{b}, i}$ and to limit the peak sensitivity degradation beyond that frequency to a factor of two $(6 \mathrm{~dB})$. If $n_{r}<n_{y}$, i. e., if there are more feedback measurements than tracked outputs, static weights with a magnitude 0.5 are used for

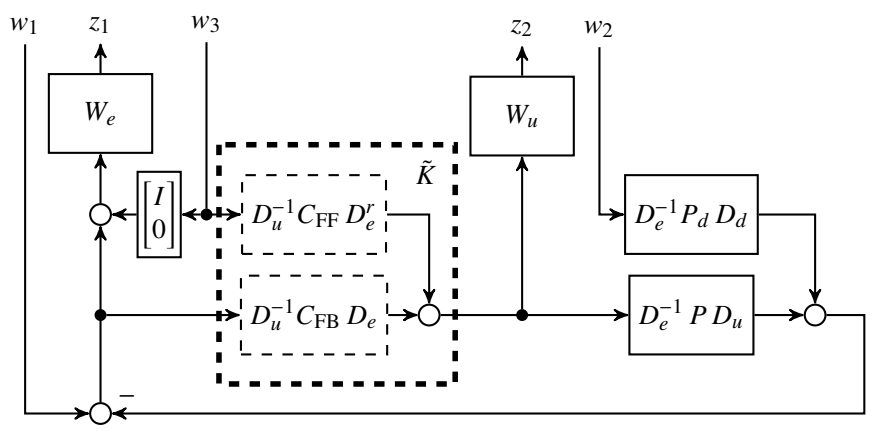

Figure 3: Generalized closed-loop interconnection for two-degrees-of-freedom design using scaled models.

the remaining $n_{y}-n_{r}$ outputs in order to limit peak sensitivity. The entries for the shaping filter $W_{u}$ are selected as first-order transfer functions with unit gain up to the available bandwidths $\omega_{\mathrm{a}, j}, j=1, \ldots, n_{u}$ of each control input and approximately differentiating behavior beyond that frequency. The result is a controller which rolls off with at least $20 \mathrm{~dB}$ per decade beyond $\omega_{\mathrm{a}, j}$ to ensure robustness and reduce noise sensitivity. The scaling matrices can be given the interpretation of maximum allowable errors $\left(D_{e}\right)$, maximum allowable inputs $\left(D_{u}\right)$, and maximum expected disturbances $\left(D_{d}\right)$. The scaling $D_{e}^{r}$ simply collects the scalings of the controlled outputs, i. e., $D_{e}^{r}=\left[\begin{array}{ll}I_{n_{r}} & 0\end{array}\right] D_{e}\left[\begin{array}{c}I_{n_{r}} \\ 0\end{array}\right]$.

\section{Automated Landing Control Problem}

The aircraft considered in this paper is representative of a twin-engined commercial transport aircraft in landing configuration (Biannic and Roos, 2015). It is described by a standard nonlinear six-degrees-of-freedom flight mechanics model with linear aerodynamics (e.g. McRuer et al., 1973) in terms of translational velocities $u, v, w$ and angular velocities $p$ (roll), $q$ (pitch), $r$ (yaw) in the body-fixed frame. Further, rate-limited first-order actuator models with saturation are included. Orientation in the earth-fixed reference frame is described in terms of Euler angles $\Phi$ (bank), $\Theta$ (pitch), and $\Psi$ (heading). The angles between body-fixed frame and wind axes are angle of attack $\alpha$ and sideslip angle $\beta$. The flight path is described with respect to earth by path angle $\gamma$, course angle $\chi$, and ground speed $V_{\mathrm{g}}$, i.e., horizontal speed relative to earth. Its orientation differs from the wind axis by the angles $\alpha_{W}$ and $\beta_{W}$ with the approximate relation (e.g. Brockhaus et al., 2013)

$$
\left[\begin{array}{c}
\beta \\
\alpha
\end{array}\right]+\left[\begin{array}{l}
\beta_{W} \\
\alpha_{W}
\end{array}\right]=\left[\begin{array}{cc}
\cos (\Phi) & \sin (\Phi) \\
-\sin (\Phi) & \cos (\Phi)
\end{array}\right]\left[\begin{array}{c}
\chi-\Psi \\
\Theta-\gamma
\end{array}\right]
$$

The final approach requires to execute a sequence of different tasks which themselves require several objectives to be fulfilled simultaneously. In the first segment of the final approach, the aircraft must track a given glide path both in vertical and horizontal direction. Hence, the deviations in vertical $(\Delta z)$ and horizontal $(\Delta y)$ direction must be controlled to zero. These deviations are available from the ILS. The standard paradigm for landing in crosswind is the "crabbed approach": The aircraft is held level $(\Phi=0)$ on the glide path and the sideslip angle $\beta$ 


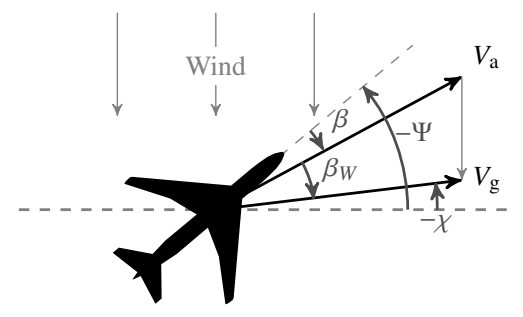

Figure 4: Attitude during crabbed approach.

is controlled to zero which according to (6) yields $\Psi \approx-\beta_{W}$. Consequently, the aircraft's nose is pointed into the wind as illustrate in Figure 4. Once the aircraft is close to the ground, sink rate is reduced for touchdown in a maneuver called "flare". The aircraft is further aligned with the direction of the runway in a second maneuver called "decrab" shortly before touchdown.

Ideally, the wings remain level throughout the whole approach and the sideslip angle is zero. Consequently, coupling of longitudinal and lateral-directional dynamics is negligible and dedicated control systems can be developed. Throttle $\left(\delta_{\text {th }}\right)$ and elevator $\left(\delta_{\mathrm{e}}\right)$ are the available actuators for longitudinal control. Lateral-directional control uses ailerons $\left(\delta_{\mathrm{a}}\right)$ and rudder $\left(\delta_{\mathrm{r}}\right)$. To facilitate the design task, a series of cascaded control systems, illustrated in Figures 5, is used.

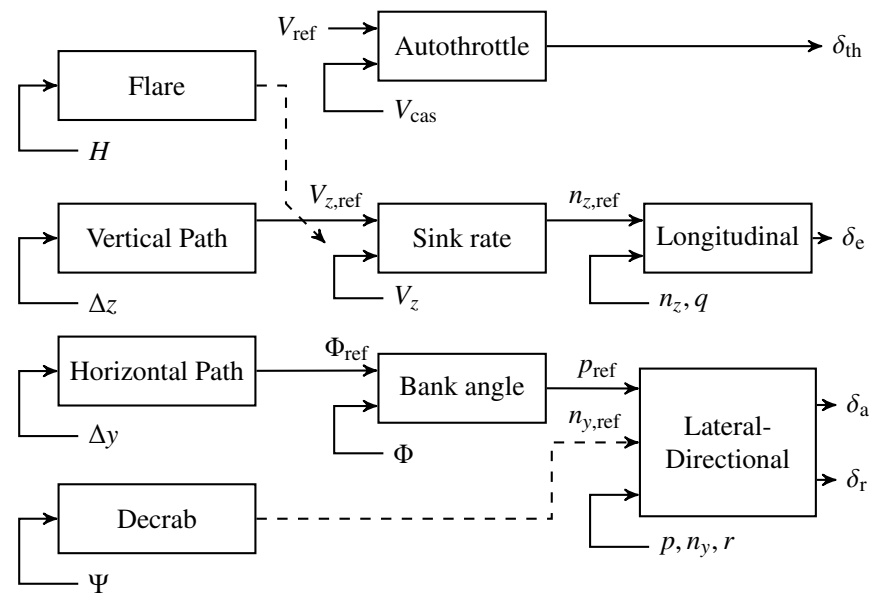

Figure 5: Control architecture for automated landing.

The cascades are arranged such that the resulting control loops resemble a chain of integrators as illustrated in Figures 6 and 7 and described in detail in Sections 3.1 and 3.2. This setup leads to a particularly simple control structure that can be designed by successive loop-closure. The bandwidth of each cascaded loop is constrained by the lower-level control loops with the ultimate constraints being the servo actuator bandwidths. The quality of the overall controller hence directly depends on the most inner control loops. Consequently, it is justified to put most effort and complexity into that part of the control system, cf. nonlinear dynamic inversion inner-loop architectures of, e. g., Looye et al. (2001) and Adams et al. (1994). In the present article, $H_{\infty}$ optimization is used to simultaneously close the most inner feedback loops. The resulting multivariable LTI controllers are much easier to implement than nonlinear dynamic inversion controllers. Using the generalized closed-loop interconnection (5), proposed in Section 2.2, these controllers are further easy to tune. All other control loops use single-input-single-output proportional or first-order LTI compensators which are designed using classical loopshaping as described in Section 2.1.

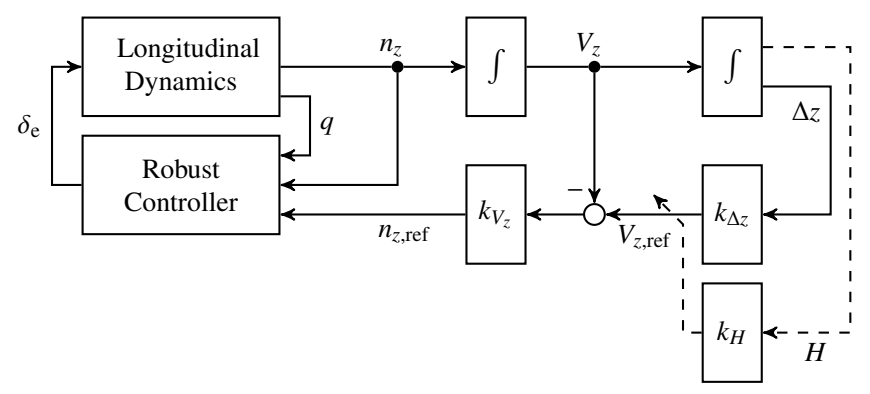

Figure 6: Integrator chain analogy for longitudinal control.

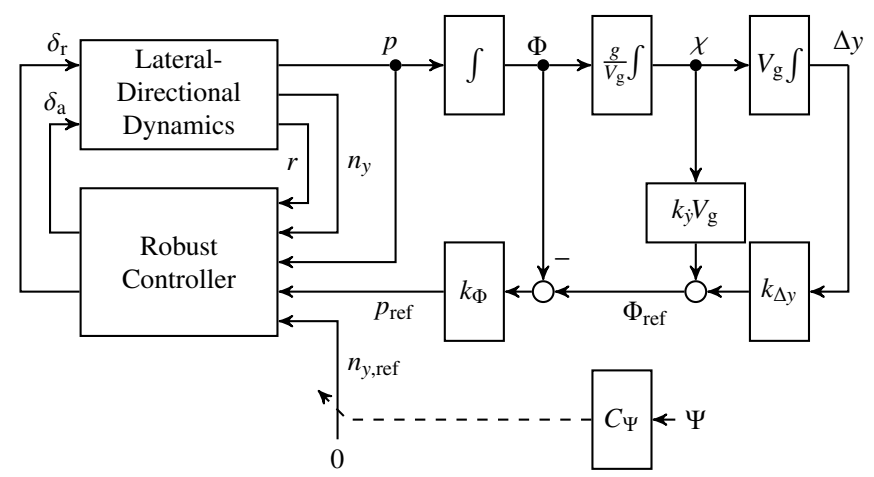

Figure 7: Integrator chain analogy for lateral-directional control.

\subsection{Longitudinal Control Architecture}

The available bandwidths for throttle and elevator differ considerably $(0.5 \mathrm{rad} / \mathrm{s}$ to $14 \mathrm{rad} / \mathrm{s})$ such that a combined control design does not promise any advantages. The setpoint $V_{\text {ref }}$ for the calibrated airspeed $V_{\text {cas }}$ is constant during final approach and it is decided to solely use throttle to control airspeed. The elevator is used to control the attitude and hence the vertical speed and position. A particularly simple architecture for this purpose is a chain of integrators as shown in Figure 6. The robust controller in the most inner feedback loop tracks the vertical acceleration $\left(n_{z}\right)$, attenuates wind disturbances, and improves short period damping with pitch rate $(q)$ measurement as an auxiliary feedback signal.

The two cascaded outer loops establish control of velocity and position, respectively. The first cascade provides a reference signal for the vertical acceleration proportional to the current sink rate $\left(V_{z}\right)$ error. The second cascade is switched according to the flight segment. During approach, the reference sink rate is generated by proportional feedback of the vertical deviation $(\Delta z)$ to keep the aircraft on the glide path. During flare, the reference sink rate is calculated proportional to the height over ground $(H)$ such that the sink rate is reduced and the aircraft touches down gently. 


\subsection{Lateral-Directional Control Architecture}

The lateral-directional control problem is necessarily multivariable and requires the coordinated use of ailerons and rudder. Thus, control design has to acknowledge crosscouplings and the different available bandwidths (16 rad/s for ailerons and $5 \mathrm{rad} / \mathrm{s}$ for rudder) such that a multivariable compensator is required.

The "crabbed approach" paradigm requires to control the sideslip angle $\beta$. For the present model, no $\beta$ measurement is available such that the lateral acceleration $n_{y}$ is used as a surrogate. Further, the vertical deviation from the glide path $(\Delta y)$ needs to be controlled with zero bank angle for zero deviation. Aiming again at building an integrator chain from bottom up, the most inner controller is designed to track a given roll rate $(p)$ and a lateral acceleration $\left(n_{y}\right)$ with yaw rate $(r)$ as an additional feedback signal to improve the lightly damped dutch roll dynamics. Cascaded control loops to track the bank angle $(\Phi)$ via roll rate command and to control the horizontal deviation from the glide path $(\Delta y)$ via bank angle command are then added. The bank angle loop simply provides a reference roll rate command proportional to the current bank angle error. The control loop for vertical deviation is a bit more involved, as the physical relation between horizontal deviation and bank angle resembles two integrator steps: Bank angle is proportional to the turn rate $\frac{\mathrm{d}}{\mathrm{d} t} \chi$ and the course angle $\chi$ is proportional to the lateral velocity $\frac{\mathrm{d}}{\mathrm{d} t} y$. Thus, proportional feedback of both lateral velocity and deviation effectively forms a proportional-derivative-type compensator. This compensator provides the bank angle reference to the lower-level control loop.

Shortly before touchdown, the aircraft is decrabbed by feeding back the heading angle $\Psi$ to provide a reference lateral acceleration. The physical relation in this case is also not strictly an integration. Hence, a dynamic compensator is required. Figure 6 shows the resulting complete lateral-directional control architecture.

\subsection{Signal Modifications}

The deviations $\Delta z$ and $\Delta y$ are obtained from the ILS glide path (GLD) and localizer (LOC) signals, respectively. These deviations are measured with respect to the receiver location on the aircraft near the cockpit which differs from the main landing gear position by $\delta x_{\mathrm{GLD}} \approx 28 \mathrm{~m}, \delta z_{\mathrm{GLD}} \approx-5 \mathrm{~m}$, and $\delta x_{\text {LOC }} \approx 30 \mathrm{~m}$ for the considered aircraft. For large heading and pitch angles that occur during landing in crosswind, the difference cannot be ignored. Consequently, the signals are corrected in order to keep the landing gear, rather than the receiver, on the glide path. Further, high frequency noise needs to be suppressed. This is achieved with complementary filters (e. g. Lambregts, 1982) to ensure minor phase loss by augmenting the signals with surrogate measurements of their respective derivatives $\frac{\mathrm{d}}{\mathrm{d} t} \Delta z \approx V_{z}$ and $\frac{\mathrm{d}}{\mathrm{d} t} \Delta y \approx V_{\mathrm{g}} \sin \chi$. The geometrically corrected and filtered deviations are

$$
\begin{aligned}
& \Delta \hat{z}=\frac{\omega_{z}}{s+\omega_{z}}\left(\left(1-\delta x_{\mathrm{GLD}} \sin \Theta\right) \Delta z+\delta z_{\mathrm{GLD}}+\frac{V_{z}}{\omega_{z}}\right), \\
& \Delta \hat{y}=\frac{\omega_{y}}{s+\omega_{y}}\left(\left(1-\delta x_{\mathrm{LOC}} \sin \Psi\right) \Delta y+\frac{V_{\mathrm{g}} \sin \chi}{\omega_{y}}\right) .
\end{aligned}
$$

The filter bandwidths are set to $\omega_{z}=2 \mathrm{rad} / \mathrm{s}$ and $\omega_{y}=0.3 \mathrm{rad} / \mathrm{s}$ based on simulations.

The sink rate over ground is different from the inertial (barometric) sink rate, if the terrain is uneven. This becomes important during flare over sloped runways, as the objective is to touch down with a specified velocity relative to the ground (cf. Lambregts, 1982). Filtering the radar altitude $H_{\mathrm{LG}}$ by an approximate differentiation filter with time constant $1 / 15 \mathrm{~s}$ yields a sufficiently accurate estimate of the landing gear's sink rate over ground

$$
\hat{V}_{z}=\frac{15 s}{s+15} H_{\mathrm{LG}}
$$

and still yields acceptable sensitivity to measurement noise.

The modified signals (7)-(8) simply replace their respective original signals in Figures 5-7. The sink rate estimate (9) replaces the barometric sink rate during flare. Throughout the remainder of this article, this replacement is implicitly assumed and the hat-notation is omitted.

\section{Design of the Inner Control Loops}

It was explained in Section 3.1 and 3.2 that the control structure is selected to resemble a chain of integrators with robust controllers at the lowest control layer. The mixed sensitivity loopshaping method of Section 2.2 requires LTI models to synthesize such $H_{\infty}$-norm optimal controllers. To obtain a model for synthesis, the nonlinear aircraft model is trimmed for quasisteady approach, i. e., constant sink rate, wings-level flight. The variety of possible load cases is considered in a grid of total mass between $120 \mathrm{t}$ and $180 \mathrm{t}$ in steps of $20 \mathrm{t}$ and center-of-mass locations between $15 \%$ and $40 \%$ length of the mean aerodynamic chord in steps of $5 \%$. Jacobian linearization with respect to each trim point is carried out to obtain a set of LTI models. These LTI models include disturbance inputs representing wind in all three directions and are used for all evaluations during the design phase.

The model with a mass of $140 \mathrm{t}$ and a center-of-mass location of $20 \%$ is used as the nominal model for synthesis. It provides a reasonable average as shown throughout Sections 4.1 and 4.2. Due to the trim condition, longitudinal and lateral-directional dynamics are decoupled which is justified for a crabbed approach as described in Section 3. Hence, the model can be split into a model of the longitudinal dynamics and a model of the lateral-directional dynamics. The robust controllers are synthesized in continuous time using Matlab's hinfsyn routine. The controllers are implemented on the nonlinear model in discrete time using standard Tustin approximation with a sampling time of $0.05 \mathrm{~s}$, i. e., a sampling rate of $20 \mathrm{~Hz}$.

\subsection{Longitudinal Robust Controller}

A short period model ( $w, q$, and elevator actuator dynamics) is used for the design of the longitudinal robust controller. The short period model approximates the system dynamics accurately above a frequency of about $0.5 \mathrm{rad} / \mathrm{s}$ as shown in Figure 8 . It is further immediately apparent that the dynamics vary only slightly for the different load cases and that the selected 
nominal model provides a reasonably accurate approximation for all load cases in the relevant frequency range. The acceleration channel exhibits a non-minimum phase zero at a frequency ranging from $2.95-3.25 \mathrm{rad} / \mathrm{s}$ for different load cases ( $3 \mathrm{rad} / \mathrm{s}$ for the nominal design model). This zero imposes a hard limitation on the achievable control bandwidth and poses a challenge for the design.
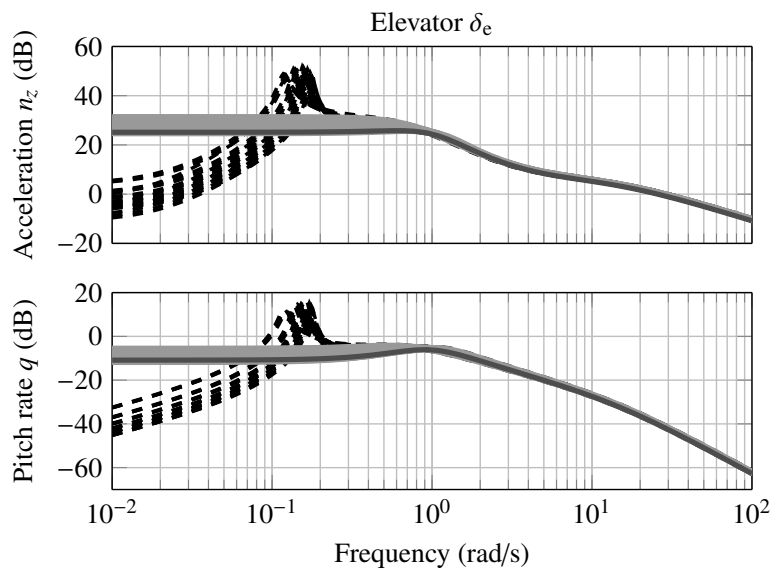

Figure 8: Longitudinal control loop complete dynamics model (- - -), short period approximation $(-)$, and nominal design model ( - ).

The design of the robust controller follows the procedure which was developed in Section 2. That is, the choice of bandwidths for the shaping filters is motivated by the discussion in Section 3 and tuning is performed by adjusting the scalings to trade off control effort versus performance. The desired bandwidth for sensitivity reduction is set to half the frequency of the non-minimum phase zero, i.e., $\omega_{\mathrm{b}, n_{z}}=1.5 \mathrm{rad} / \mathrm{s}$. The available control bandwidth limitation is set to half the actuator bandwidth, i. e., $\omega_{\mathrm{a}, \delta_{\mathrm{e}}}=7 \mathrm{rad} / \mathrm{s}$. The available control effort is selected in terms of the input scaling as $D_{u, \delta_{\mathrm{e}}}=30^{\circ}$. Next, the ratio of the scalings for $n_{z}$ and $q$ is fixed as $10 \mathrm{~m} / \mathrm{s}^{2}$ to $15^{\circ} / \mathrm{s}$, which resembles the steady-state response of the open-loop model when excited by a step input with size $D_{u, \delta_{\mathrm{e}}}$. Subsequently, these values are multiplied by a common scalar for tuning. Lower values increase the control effort as the ratio between "maximum error" and "maximum control effort" is changed. Similarly, the "maximum disturbance" $D_{d}$, representing vertical wind, is used for tuning. It directly affects the short period damping, such that the choice can be guided by the response characteristic and the resulting closed-loop pole locations. For values of $D_{d}=5 \mathrm{~m} / \mathrm{s}$, $D_{e, n_{z}}=0.7 \mathrm{~m} / \mathrm{s}^{2}$, and $D_{e, q}=1.05^{\circ} / \mathrm{s}$ a satisfactory controller is obtained which leads to consistent acceleration responses across all models with a damping ratio of about 0.7 .

The resulting multivariable controller is of fifth order and maps $n_{z}(\mathrm{~m} / \mathrm{s})$ and $q(\mathrm{rad} / \mathrm{s})$ to $\delta_{\mathrm{e}}(\mathrm{rad} / \mathrm{s})$. Its frequency response is provided in Figure 9. It resembles a conventional PI compensator and pitch damper configuration, but provides additional lead compensation and roll-off in both channels. Further, the feedforward gain of the acceleration signal is about $6 \mathrm{~dB}$ larger than the feedback gain.

The frequency responses of the sensitivity functions, calculated for all 24 load cases using the complete longitudi-
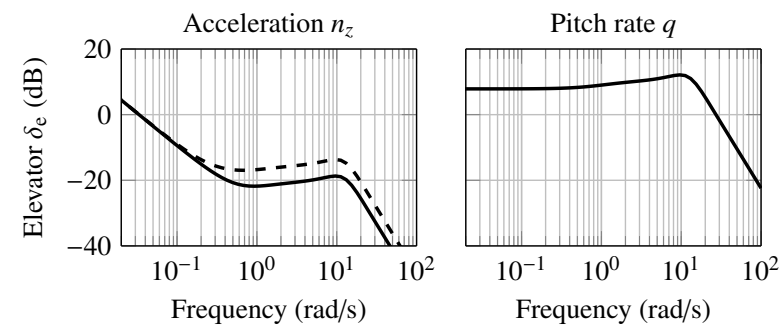

Figure 9: Longitudinal robust controller (feedforward - - - / feedback -

nal dynamics models, are shown in Figure 10. The tracking bandwidth, represented by the reference transmission function $R=S P C_{\mathrm{FF}}$, is around $1.5 \mathrm{rad} / \mathrm{s}$ and the sensitivity reduction below $1.5 \mathrm{rad} / \mathrm{s}$ is particularly apparent in the disturbance sensitivity shown in Figure 10b. Further, sensitivity degradation is confined to the frequency range between 1 and $10 \mathrm{rad} / \mathrm{s}$ with a peak of about $3 \mathrm{~dB}$, indicating good robustness.
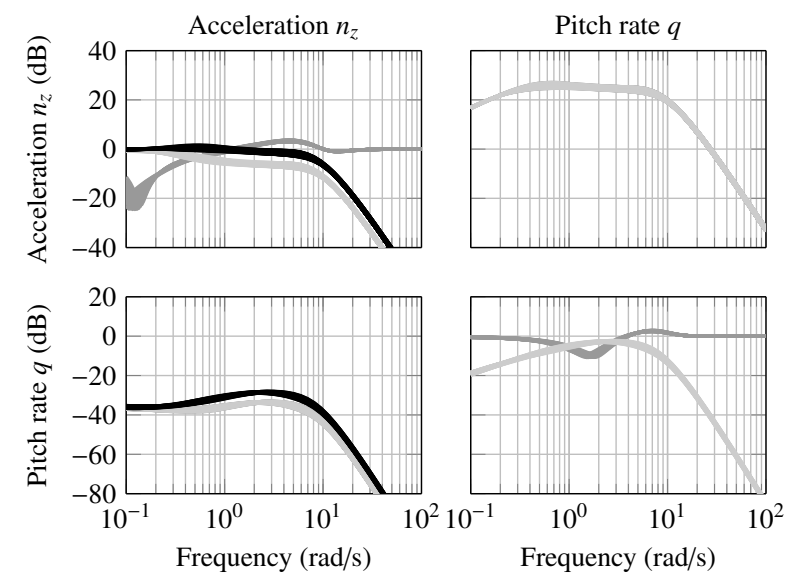

(a) Sensitivity $(-)$, complementary sensitivity $(-)$, and reference transmission ( - )

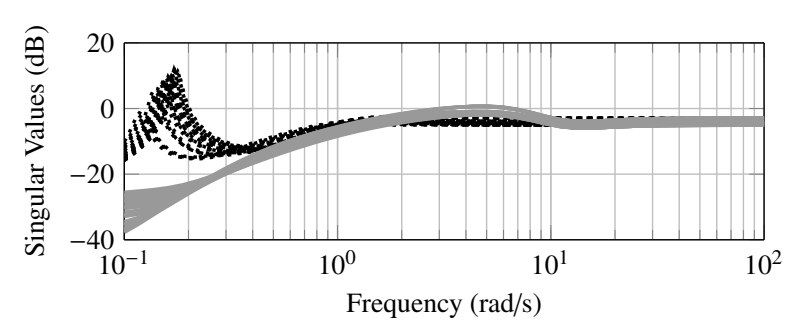

(b) Sensitivity of vertical acceleration $n_{z}$ to vertical and horizontal wind disturbances for open loop (-- -) and closed loop ( - ).

Figure 10: Longitudinal control loop.

\subsection{Lateral-Directional Control}

Similar to the longitudinal case, an approximation of the fast dynamics is used to design the lateral-directional controller. For the lateral-directional model, the fast dynamics consist of the dutch roll mode, the roll subsidence mode, and actuator dynamics. The resulting approximation, shown in Figure 11, omits the slow spiral mode and captures the dynamics very accurately above a frequency of about $0.5 \mathrm{rad} / \mathrm{s}$. Again, the variation over different load cases is marginal. 

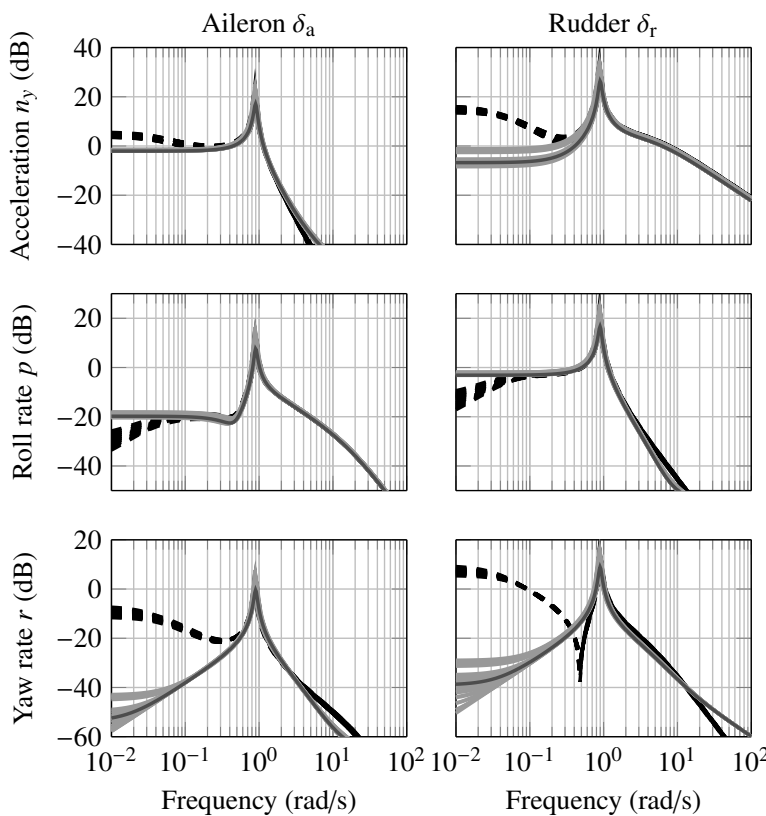

Figure 11: Lateral-directional control loop complete dynamics model (- - -), approximation ( $(-)$, and nominal design model ( $\square$ ).

The generalized interconnection (5) and the procedure from Section 2 are used to design a robust controller. The desired bandwidths for sensitivity reduction are set to $\omega_{\mathrm{b}, n_{y}}=0.5 \mathrm{rad} / \mathrm{s}$ for the lateral acceleration and $\omega_{\mathrm{b}, p}=1.5 \mathrm{rad} / \mathrm{s}$ for the roll rate. The available actuator bandwidths are set to half the actuator bandwidths, i.e., $\omega_{\mathrm{a}, \delta_{\mathrm{a}}}=8 \mathrm{rad} / \mathrm{s}$ for the aileron and $\omega_{\mathrm{a}, \delta_{\mathrm{r}}}=2.5 \mathrm{rad} / \mathrm{s}$ for the rudder. The scalings are used for tuning. Roll-yaw coupling is adjusted via the ratio of the scalings for $n_{y}$ and $p$ and damping is addressed using the scaling for $r$. A choice of maximum allowable errors of $D_{e, n_{y}}=0.2 \mathrm{~m} / \mathrm{s}^{2}$, $D_{e, p}=1 \%$, and $D_{e, r}=1 \% \mathrm{~s}$ in the matrix $D_{e}$ is made. The maximum magnitude for lateral gust disturbances is selected as $D_{d}=5 \mathrm{~m} / \mathrm{s}$. Finally, the scalings for maximum control signals are tuned to $D_{u, \delta_{\mathrm{a}}}=15^{\circ}$ aileron and $D_{u, \delta_{\mathrm{r}}}=30^{\circ}$ rudder to remain inside the actuator limits.

The resulting multivariable controller is of ninth order. Figure 12 shows its frequency response. Again, additional lead compensation and roll-off characteristics are apparent features compared to a conventional control configuration. Similarities are, e.g., the damping augmentation through yaw-rateto-rudder feedback and a pronounced roll-rate-command-torudder forward feed to cancel adverse yaw.

The frequency responses of the corresponding sensitivity functions are shown in Figure 13. The sensitivity in the rollrate channel is reduced below the desired frequency of $1.5 \mathrm{rad} / \mathrm{s}$ and the peak is less than $3 \mathrm{~dB}$. The specifications for the lateralacceleration channel are not completely achieved and the sensitivity is reduced only below a frequency of about $0.2 \mathrm{rad} / \mathrm{s}$. The sensitivity peak in this channel is about $6 \mathrm{~dB}$ and hence inside the specified factor-of-two bound. Disturbance rejection is clearly improved around the frequency of the dutch roll mode $(0.9 \mathrm{rad} / \mathrm{s})$ with well-behaved degradation towards lower frequencies. The feedforward part of the controller improves decoupling between $n_{y}$ and $p$.
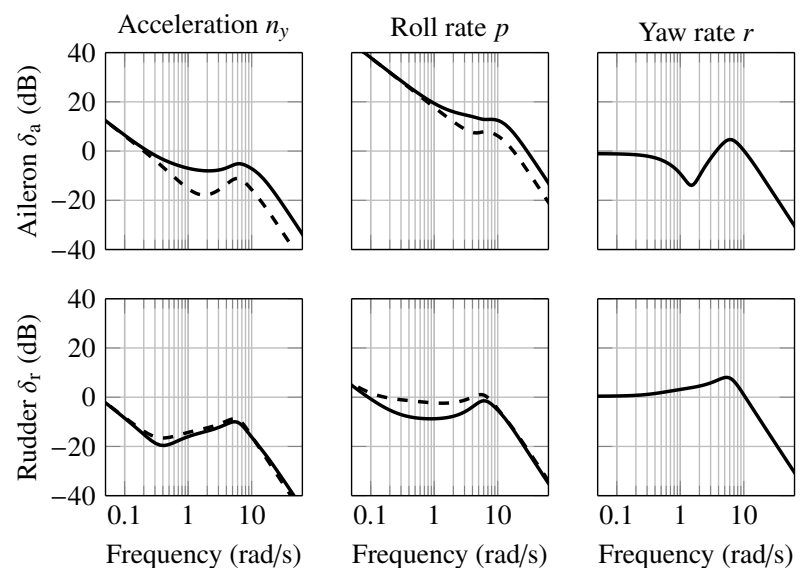

Figure 12: Lateral-directional robust controller (feedforward - - - / feedback -)
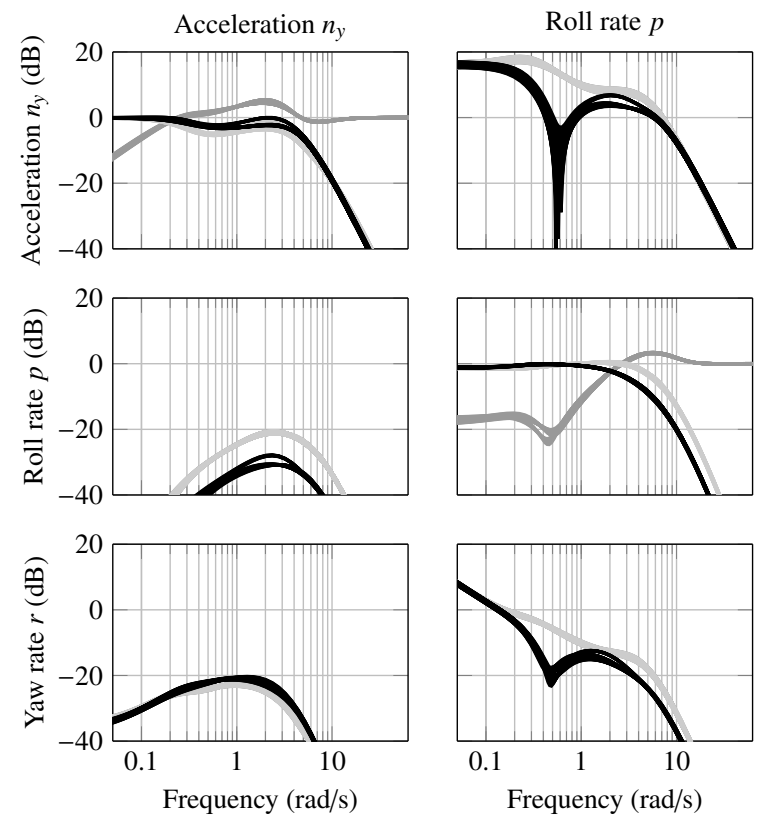

(a) Sensitivity $(-)$, complementary sensitivity $(-)$, and reference transmission ( - ).

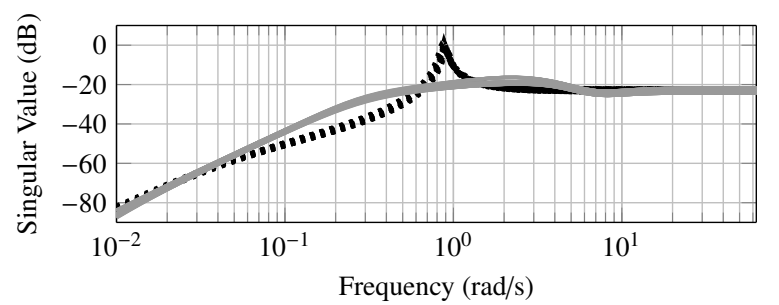

(b) Sensitivity to lateral wind disturbances for open loop (- - -) and closed loop (-).

Figure 13: Lateral-directional control loop.

\section{Design of the Outer Control Loops}

With the inner controllers providing tracking capabilities, the remaining outer loops can be designed through successive loopclosure to provide reference signals for the lower-level controllers. All outer loops are designed using classical loopshaping such that the looptransfer functions of all 24 models are 
close to a target loopshape. These target loopshapes are chosen with crossover frequencies of at least a factor of 2 below the control loop bandwidth of the respective lower-level control loop. Each individual loop satisfies robustness margins of at least $8 \mathrm{~dB}$ gain and $50^{\circ}$ phase. The latter also provides robustness against feedback delays.

\subsection{Autothrottle}

The longitudinal robust controller provides attitude stabilization and the low engine bandwidth of $0.5 \mathrm{rad} / \mathrm{s}$ makes it impossible to counteract turbulence through autothrottle. Hence, the only purpose of the autothrottle loop is to maintain airspeed in varying average wind conditions. A controller is designed such that a target loopshape with a bandwidth of $0.25 \mathrm{rad} / \mathrm{s}$ as shown in Figure 14 is approximately achieved for all 24 linearized models. This loopshaping approach results in a PI controller $\delta_{\text {th }}=0.045\left(1+\frac{1}{15} \frac{1}{s}\right)\left(V_{\text {ref }}-V_{\text {cas }}\right)$.

The sensitivity functions, calculated with the complete longitudinal model including the longitudinal robust controller, are also shown in Figure 14. They confirm that sensitivity is reduced below a frequency of about $0.2 \mathrm{rad} / \mathrm{s}$ and indicate well behaved sensitivity degradation above that frequency.

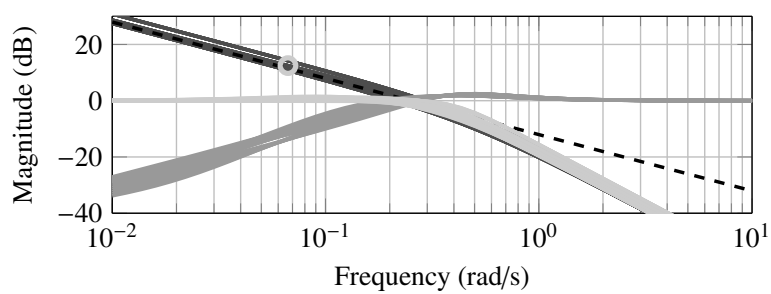

(a) Looptransfer ( $\longrightarrow$ ), target loop shape (- - -), compensator zero $(\bigcirc)$, sensitivity $(-)$, and complementary sensitivity $(-)$.

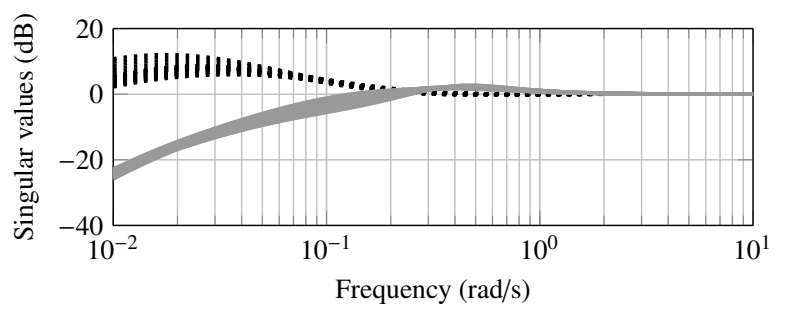

(b) Airspeed sensitivity to vertical and horizontal wind disturbances for open loop (- - -) and closed loop ( - ).

Figure 14: Autothrottle control loop.

\subsection{Sink Rate Control}

Vertical acceleration is the controlled output in the longitudinal inner control loops. Hence, the transfer function from sink rate to vertical acceleration closely resembles an integrator. The target loopshape for this control loop is an integrator with a crossover frequency of less than half the tracking bandwidth of the $n_{z}$ loop $(1.5 \mathrm{rad} / \mathrm{s})$ in order to ensure sufficient phase margin. Hence, the maximum crossover frequency across all load cases should be below $0.75 \mathrm{rad} / \mathrm{s}$, which translates to a minimum crossover of about $0.6 \mathrm{rad} / \mathrm{s}$ as shown in Figure 15. A proportional compensator $n_{z \text {,ref }}=k_{V_{z}}\left(V_{z \text {,ref }}-V_{z}\right), k_{V_{z}}=0.625$ is used to adjust the crossover frequency accordingly. The sensitivity functions, calculated with the complete longitudinal model including the inner controller, confirm sensitivity reduction below $0.6 \mathrm{rad} / \mathrm{s}$ with a sensitivity peak of $6 \mathrm{~dB}$. The $n_{z}$ reference signal provided to the inner controller is further limited to $\pm 5 \mathrm{~m} / \mathrm{s}^{2}$ to ensure safe operation.

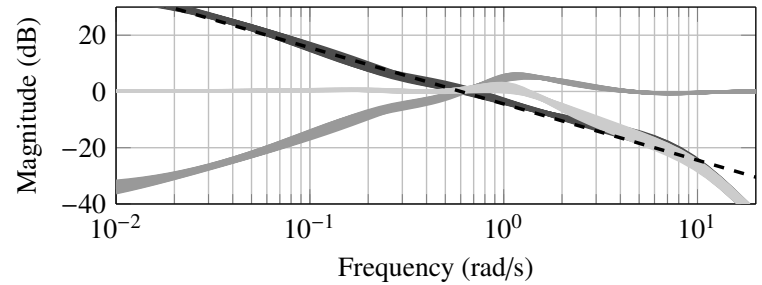

(a) Looptransfer ( $\longrightarrow$ ), target loop shape (- - -), sensitivity ( $\square$ ), and complementary sensitivity (-).

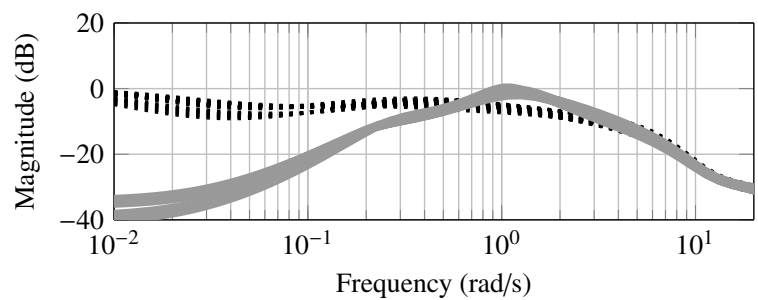

(b) Sink rate sensitivity to wind disturbances for open loop (- - -) and closed loop ( $)$.

Figure 15: Sink rate control loop

\subsection{Bank Angle Tracking}

The transfer function from roll rate to bank angle also resembles an integrator, as the roll rate is one of the controlled outputs in the lateral-directional inner controller. Hence, a proportional compensator $p_{\text {ref }}=k_{\Phi}\left(\Phi_{\text {ref }}-\Phi\right)$ with $k_{\Phi}=0.7$ is sufficient to achieve the target loopshape with a crossover frequency of $0.7 \mathrm{rad} / \mathrm{s}$ (half the tracking bandwidth of the roll rate loop) as shown in Figure 16a. Figure 16b shows the resulting sensitivity to wind disturbances.

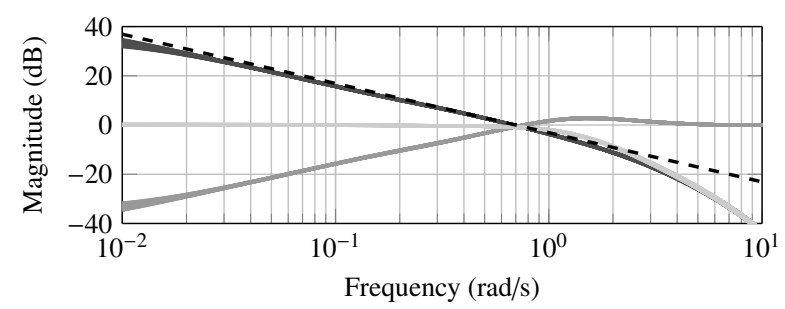

(a) Looptransfer ( $\square$ ), target loopshape (- - -), sensitivity ( $\square$ ), and complementary sensitivity ( $)$.

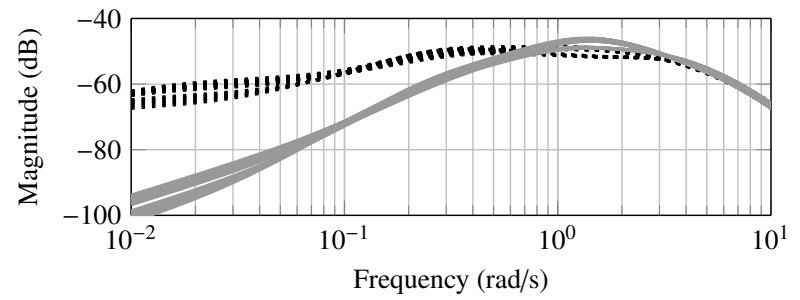

(b) Bank angle sensitivity to lateral wind disturbances for open loop (- - -) and closed loop ( $)$.

Figure 16: Bank angle control loop. 


\subsection{Vertical Glide Path Tracking}

As an immediate consequence of the sink rate control loop, the transfer function from $V_{z}$ to the vertical glide path deviation $\Delta z$ is also an integrator in the frequency range of interest. Hence, a proportional compensator $V_{z, \text { ref }}=k_{\Delta z} \Delta z$ with $k_{\Delta z}=0.1$ is sufficient to set a closed-loop bandwidth of $0.1 \mathrm{rad} / \mathrm{s}$ as shown in Figure 17a. This relatively low bandwidth was selected to avoid unnecessary control activity during approach but is still sufficiently high to ensure tracking and improve disturbance rejection as shown in Figure $17 \mathrm{~b}$. To ensure safe operation, the output of the controller is further limited to sink rate commands that deviate $\pm 3 \mathrm{~m} / \mathrm{s}$ from the trim value.

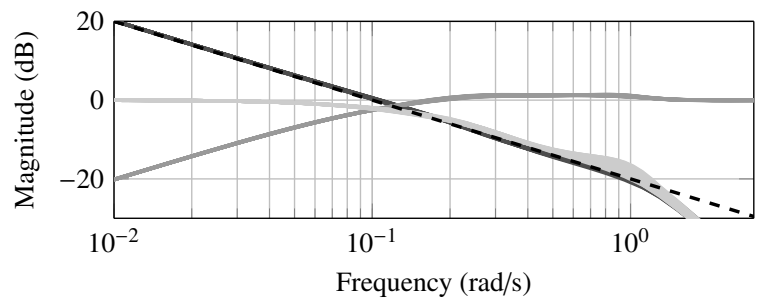

(a) Looptransfer ( $\square$ ), target loopshape (- - -), sensitivity ( $\square$ ), and complementary sensitivity $(-)$.

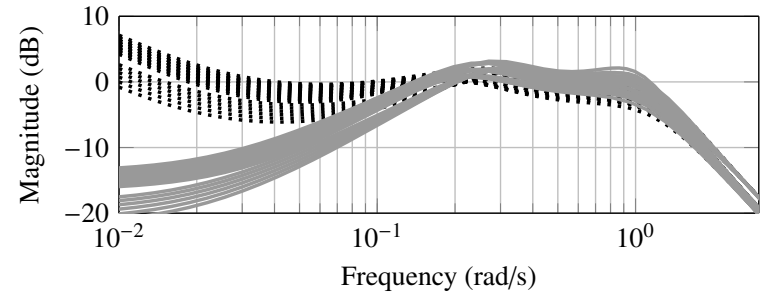

(b) Vertical glide path deviation sensitivity to vertical wind disturbances for open loop (- - - ) and closed loop ( - ).

Figure 17: Vertical glide path deviation control loop.

\subsection{Horizontal Glide Path Tracking}

As described in Section 3.2, the transfer function from $\Phi$ to $\Delta y$ resembles a double integrator in the relevant frequency range once control over $\Phi$ is established. Thus, additional phase lead is required to stabilize the loop and a proportional-derivative controller is designed to achieve a bandwidth of $0.3 \mathrm{rad} / \mathrm{s}$ (half the tracking bandwidth of the bank angle loop) and to match the target loopshape over 1 decade around crossover. Differentiation is not actually performed, but instead the lateral velocity estimate $\frac{\mathrm{d}}{\mathrm{d} t} \Delta y \approx V_{\mathrm{g}} \sin \chi$ is used for feedback once the controller is implemented (cf. Figure 7). The controller equation is $\Phi_{\text {ref }}=k_{\Delta y} \Delta y+k_{\dot{y}} V_{\mathrm{g}} \sin \chi$ with $k_{\Delta y}=0.003$ and $k_{\dot{y}}=0.033$. The bank angle command is limited to $\pm 30^{\circ}$ to ensure safe operation.

\subsection{Flare}

Flare is engaged at a fixed altitude $H_{0}=20 \mathrm{~m}$ above ground and the autothrottle is deactivated. Ideally, the time series of the height over ground should follow an exponentially decaying trajectory $H(t)=\left(H_{0}+H_{\text {bias }}\right) \mathrm{e}^{-\frac{t}{\tau}}-H_{\text {bias }}$, where $H_{0}$ is the altitude

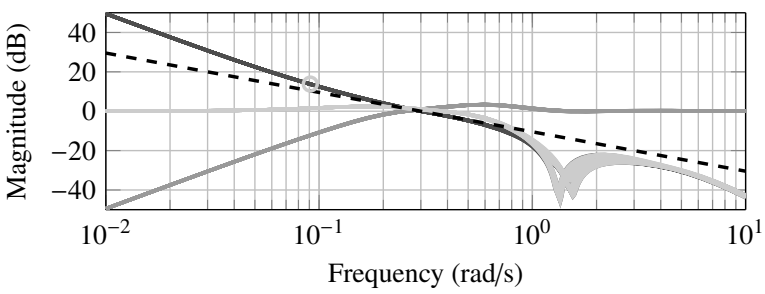

(a) Looptransfer (-), target loopshape (- - -), compensator zero $(\bigcirc)$, sensitivity $(-)$, and complementary sensitivity $(-)$.

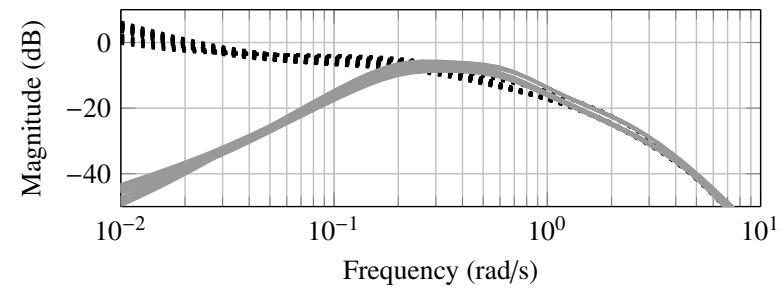

(b) Lateral glide path deviation sensitivity to lateral wind disturbances for open loop (-- -) and closed loop ( $(-)$.

Figure 18: Horizontal glide path deviation control loop.

at flare initialization and $H_{\text {bias }}>0$ adjusts the point of touchdown (Lambregts and Creedon, 1980). This trajectory is exactly attained when $\dot{H} \equiv-1 / \tau\left(H+H_{\text {bias }}\right)$. Since $\dot{H}=-V_{z}$, an equivalent representation for this condition is $V_{z} \equiv 1 / \tau\left(H+H_{\text {bias }}\right)$. Thus, closing a feedback loop $V_{z, \text { ref }}=k_{H}\left(H+H_{\text {bias }}\right)$ from radar altitude to sink rate reference establishes the desired behavior. The proportional gain of this feedback law is $k_{H}=1 / \tau$ and the offset $H_{\text {bias }}$ is calculated such that the sink rate at flare initialization matches the sink rate during approach. That is, $H_{\text {bias }}=\tau V_{z \text {,Approach }}-H_{0}$. The time constant is calculated as

$$
\tau=H_{0} /\left(V_{z, \text { Approach }}-V_{z, \text { Touchdown }}\right)
$$

to result in a touchdown velocity $V_{z \text {,Touchdown }}=0.3 \mathrm{~m} / \mathrm{s}(1 \mathrm{ft} / \mathrm{s})$.

The constants $H_{\text {bias }}$ and $\tau$ are determined when flare is activated using $V_{z \text {,Approach }}:=\frac{5}{s+5} V_{z \text {,ref }}$ as an estimate for the current sink rate. Doing so accounts for wind disturbances and different approach velocities of the aircraft and leads to a "variable tau" control law, similar to the one proposed by Lambregts and Creedon (1980). The time constant (10) turns out to vary between $4-8 \mathrm{~s}$, which translates to a gain $k_{H}$ between 0.125 and 0.25 . The maximum bandwidth of the resulting control loop is at most $0.25 \mathrm{rad} / \mathrm{s}$. The sink rate loop with its minimum bandwidth of $0.6 \mathrm{rad} / \mathrm{s}$ is hence sufficiently separated and requires no further adjustment.

\subsection{Decrab}

Decrab is activated at a fixed altitude of $5 \mathrm{~m}$ above ground. A lag compensator $C_{\Psi}=33 \frac{4 s+1}{20 s+1}$ is designed to close the feedback loop $n_{y \text {,ref }}=-C_{\Psi} \Psi$ such that the target loopshape of Figure 19 with a crossover frequency of $0.6 \mathrm{rad} / \mathrm{s}$ is achieved. This bandwidth was selected to remain inside the actuator limits during decrab. Bank angle is still controlled through the glide path deviation control loop during decrab to keep the flight path aligned with the centerline. The limits on bank angle commands are, however, tightened to $\pm 5^{\circ}$ in order to ensure suf- 
ficiently level wings for touchdown. No additional wings-level command is issued.

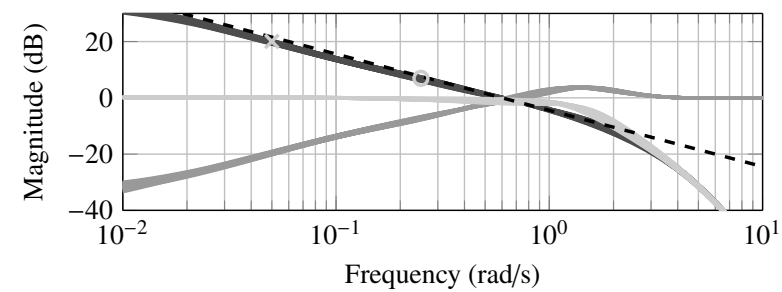

(a) Looptransfer (-), target loopshape (- - ), compensator pole/zero $(\times / 0)$, sensitivity $(-)$, and complementary sensitivity $(-)$.

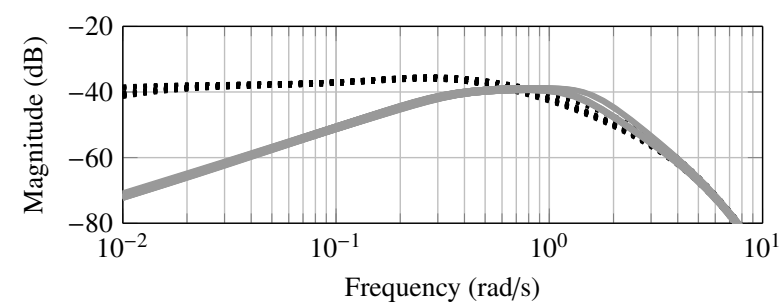

(b) Heading sensitivity to lateral wind disturbances for open loop (- - -) and closed loop ( - ).

Figure 19: Heading angle control loop for decrab.

\section{Design Verification}

The autolanding system is verified in the nonlinear simulation for final approach and landing ${ }^{1}$. This simulation environment considers wind shear, atmospheric turbulence, and also acknowledges ground effects. It includes an ILS model that provides vertical and horizontal deviations from the glide path. These signals are corrupted by measurement noise and their accuracy depends on the distance to the runway.

Figure 20a shows the final phase of a simulated landing in time-varying but non-turbulent crosswind. The evaluation criteria are the risk of short landing, long landing, hard landing, decentered landing, landing with steep bank angle, and landing with steep wheel sideslip angle as defined by Biannic and Boada-Bauxell (2016). The upper left plot depicts the main landing gear's altitude $H_{\mathrm{LG}}$ above ground. The altitude $60 \mathrm{~m}$ after the runway threshold is marked with a square and must be positive to satisfy the short landing criterion. The altitudes for flare and decrab initialization are visualized by dashed lines. The touchdown point evaluated for the long landing criterion is marked with a diamond. The exponential flare trajectory is clearly visible. The upper right plot shows the sink rate above ground. The touchdown speed for the hard landing criterion is marked with a triangle and the maximum value of $10 \mathrm{ft} / \mathrm{s}$ $(3.05 \mathrm{~m} / \mathrm{s})$ is indicated by the arrow on the right. In the present simulation, the aircraft touches down gently, with a velocity of approximately $1 \mathrm{~m} / \mathrm{s}$. The lower left plot shows a view on the runway from above with the centerline highlighted. The trajectory of the aircraft closely follows the centerline all the way

${ }^{1}$ CALC package version 7 released on February 7th 2017, openly available from http://w3.onera.fr/smac/?q=aircraftModel through flare and decrab. The touchdown point determines the decentered landing criterion and is marked with a cross. Arrows on the right illustrate the maximum admissible deviation of $\pm 15 \mathrm{~m}$ from the centerline. Bank angle and wheel sideslip are shown in the lower right plot and the values at touchdown that determine the steep bank angle and steep wheel sideslip criteria are marked by circles. The aircraft approaches crabbed with a heading of about $-10^{\circ}$ and zero bank angle. Once decrab is initiated, heading is quickly reduced to $0^{\circ}$ with only minor effect on the bank angle. The admissible range is again marked by arrows on the right.

The same set of parameters is used for a second simulation which additionally includes turbulence in Figure 20b for comparison. The flare maneuver again reduces the sink rate to about $1 \mathrm{~m} / \mathrm{s}$ at touchdown, although the strong gust disturbances remain clearly visible in the response. The influence of turbulence on the other evaluation criteria is negligible and the aircraft performs qualitatively identical to the non-turbulent scenario, confirming the controller's ability to handle turbulent wind conditions. Figure 21 shows the corresponding time series of the simulation run with turbulence and also includes the approach segment. The aircraft requires roughly $20 \mathrm{~s}$ to initially acquire the glide path and then tracks it very tightly. An increasing crosswind component causes the aircraft to build up a yaw angle of $-10^{\circ}$ during approach. The functionality of the cascaded control system is confirmed, with lower-level loops clearly tracking the reference signals from their respective higher-level loops. Comparing the plot of the vertical deviation from the glide path in Figure $21 \mathrm{~b}$ with the plot of the vertical deviation from the centerline in Figure 20 shows that the tracking error is the result of a localizer bias in this particular simulation. Finally, control surface usage is well within the saturation limits with the decrab maneuver clearly requiring the most control effort.

The six criteria are next evaluated in Monte Carlo simulations to verify robustness across all possible load cases and a variety of environmental parameters. First, 100000 simulations with random parameters, distributed according to Table 1 (Biannic and Boada-Bauxell, 2016), are performed to determine the average risk dispersion. In a second step, another 100000 simulation trials are performed to determine the limit risk dispersion. In this case, crosswind is fixed to 25 knots right throughout all simulation trials while all other parameters again follow Table 1. One simulation trial takes about 2 seconds such that the two Monte Carlo simulation campaigns took 5 days computation time on a standard desktop PC. Figure 22 shows histograms of the Monte Carlo simulation campaigns. Fixing the crosswind to its maximum value mostly affects the steep bank angle and steep wheel sideslip criteria but also results in larger vertical speeds at touchdown.

Figure 23 shows the resulting risk dispersions as cumulative distribution functions for each of the six evaluation criteria. The requirements are illustrated by the shaded areas. The distribution function has to be completely outside of these areas for average risk. For limit risk, it is allowed to penetrate the lightly shaded areas, but must remain outside the darker areas. The $d e-$ centered landing criterion, for example, requires the probability for a lateral deviation of greater than $15 \mathrm{~m}$ from the centerline 

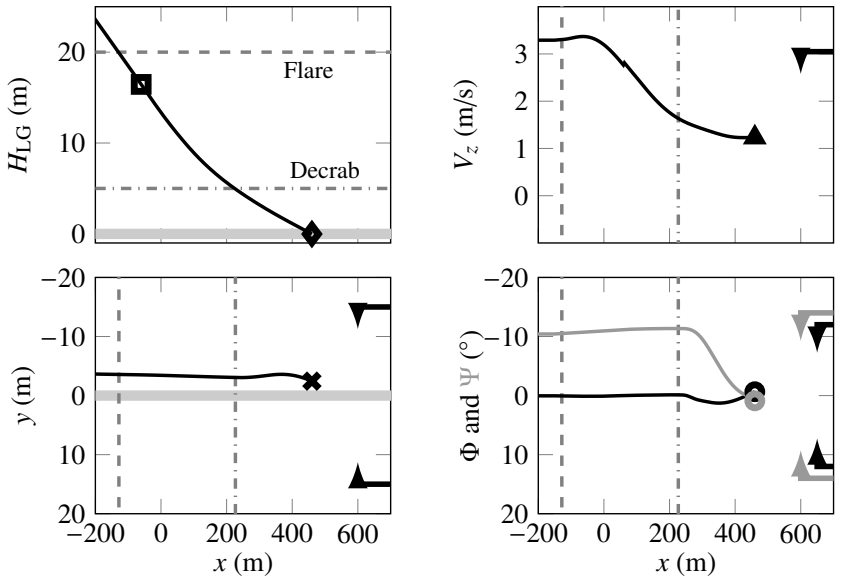

(a) Nominal simulation with 25 knots non-turbulent crosswind.
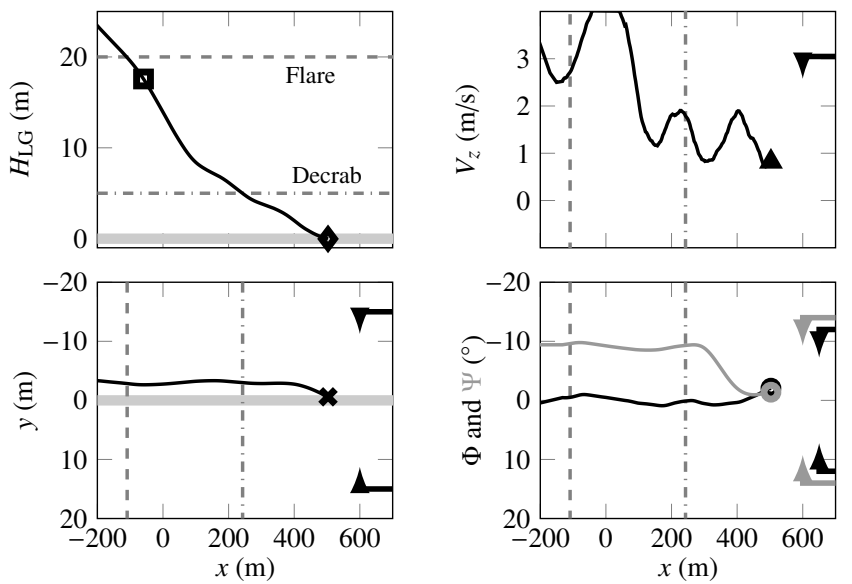

(b) Simulation with 25 knots turbulent crosswind.

Figure 20: Nonlinear simulation of of crosswind landing with marks for evaluation criteria: $H_{60}(\boldsymbol{\square}), X_{\mathrm{T}}(\boldsymbol{\diamond}), V_{z, \mathrm{~T}}(\boldsymbol{\Delta}), Y_{\mathrm{T}}(\mathbf{X}), \Phi_{\mathrm{T}}(\mathbf{0})$, and $\Psi_{\mathrm{T}}(0)$.

Table 1. Monte Carlo Parameters for Verification.

\begin{tabular}{lccc}
\hline Parameter & distribution* & min & $\max$ \\
\hline Lateral wind (knots) & $\mathcal{N}(0,7)$ & 25 left & 25 right \\
Longitudinal wind (knots) & $\mathcal{N}(7.5,7.5)$ & 10 tail & 30 head \\
Mass $(\mathrm{t})$ & uniform & 120 & 180 \\
Center of mass $(\%)$ & uniform & 15 & 41 \\
Temperature $\left({ }^{\circ} \mathrm{C}\right)$ & uniform & -69 & 40 \\
Runway slope $(\%)$ & $\mathcal{N}(0,0.4)$ & -2 & 2 \\
Glide slope $\left({ }^{\circ}\right)$ & $\mathcal{N}(-3,0.075)$ & -2.85 & -3.15 \\
Localizer bias $(\mu \mathrm{A})$ & $\mathcal{N}(0,2.5)$ & -5 & 5 \\
Runway altitude $(\mathrm{ft})$ & {$[-1000,250]: 50.00 \%$} & -1000 & 9200 \\
& {$[250,750]: 28.33 \%$} & & \\
& {$[750,1250]: 13.33 \%$} & & \\
& {$[1250,1750]: 3.33 \%$} & & \\
& {$[1750,2500]: 1.67 \%$} & & \\
& {$[2500,3500]: 1.00 \%$} & & \\
& {$[3500,4500]: 0.67 \%$} & & \\
& {$[4500,9200]: 1.67 \%$} & & \\
\hline
\end{tabular}

*N $(\mu, \sigma)$ : normal distribution with mean $\mu$ and standard deviation $\sigma$.
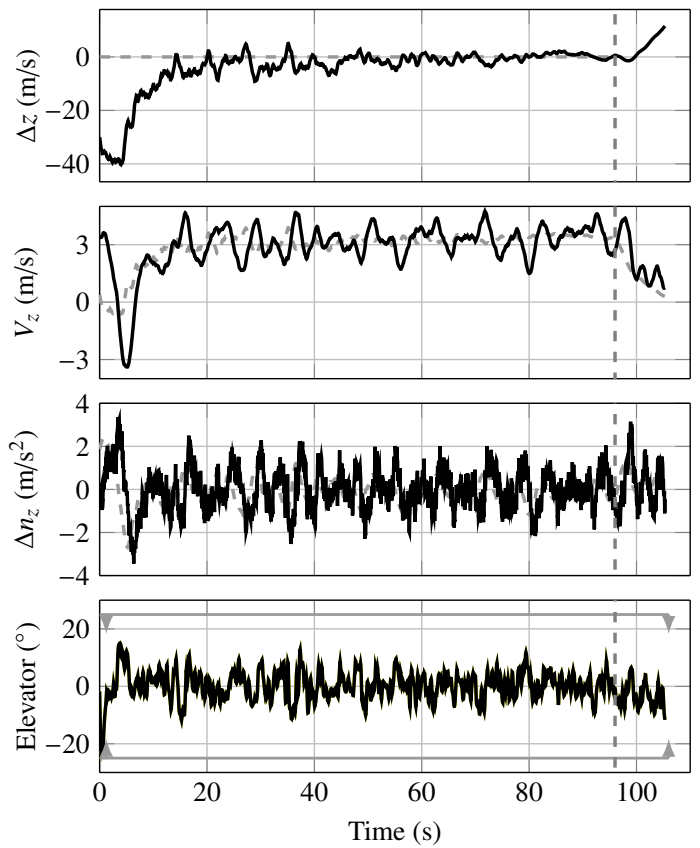

(a) Longitudinal control loops.
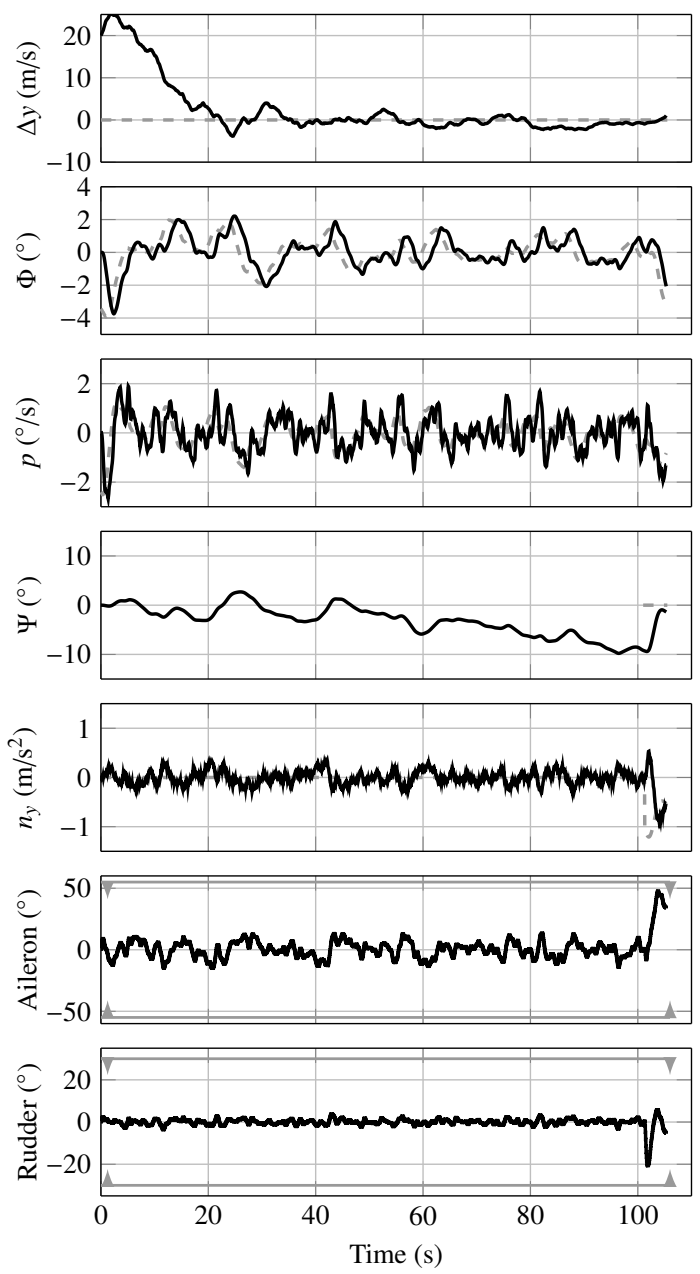

(b) Lateral-directional control loops.

Figure 21: Nonlinear simulation with 25 knots turbulent crosswind (references $-=-$ / actual values - ). 

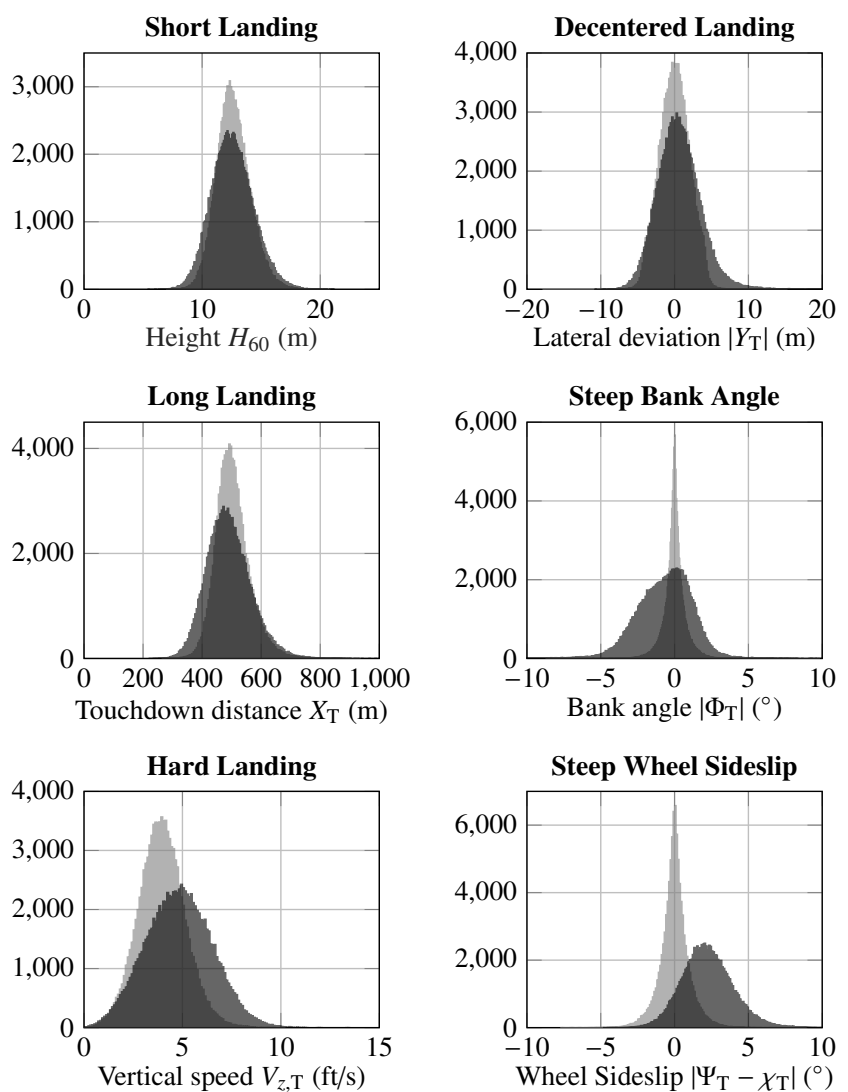

Figure 22: Histograms of average risk $(\square)$ and limit risk $(\square)$ evaluation campaign, each obtained from 100000 Monte Carlo simulations.

at touchdown to be less than $10^{-6}$ for average risk and less than $10^{-5}$ for limit risk evaluation.

All criteria are satisfied for 25 knots maximum crosswind by the design. The only limiting factor is the hard landing criterion, while all other criteria show generous margins. Hard landing can be attributed to insufficiently tight sink rate control during flare. Improving this is however not easy, as the nonminimum phase zero imposes a hard constraint on the achievable bandwidth of the control loop.

\section{Conclusion}

A comprehensive autopilot design for crosswind landings was presented. The selection of a suitable cascaded control architecture resembling chains of integrators, necessary signal modifications, and the design of all control loops were detailed. The proposed mixed sensitivity formulation uses design parameters with a clear relation to the design requirements so that little tuning effort was required. The control system was evaluated in nonlinear Monte Carlo simulations and shown to successfully land the aircraft in 25 knots turbulent crosswind.

\section{References}

Adams, R. J., Buffington, J. M., Sparks, A. G., Banda, S. S., 1994. Robust Multivariable Flight Control. Springer, London.
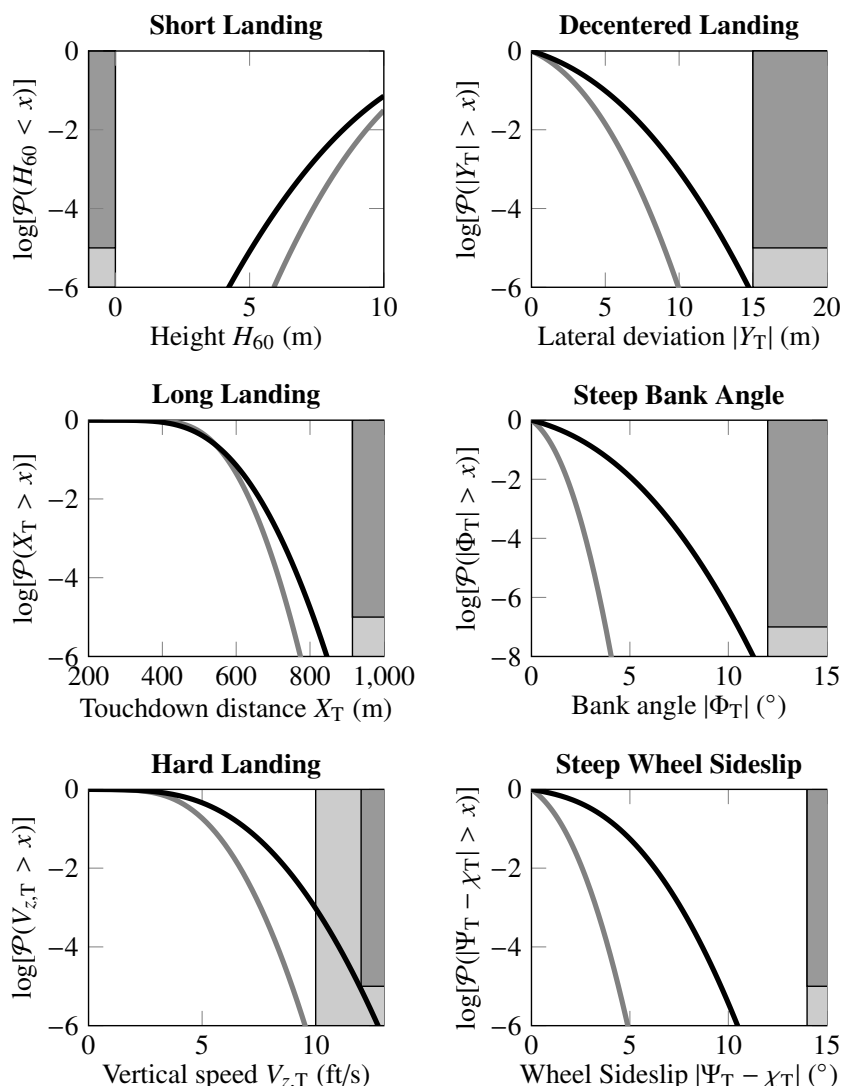

Figure 23: Dispersions for average ( - ) and limit risk ( - ), each obtained from 100000 Monte Carlo simulations. The shaded areas represent the average risk $(\square)$ and limit risk $(\square)$ criteria.

Biannic, J., Apkarian, P., 2001. A new approach to fixed-order $H_{\infty}$ synthesis: Application to autoland design.

Biannic, J., Roos, C., 2015. Flare control law design via multi-channel $H_{\infty}$ synthesis: Illustration on a freely available nonlinear aircraft benchmark. In: American Control Conference. pp. 1303-1308.

Biannic, J.-M., Boada-Bauxell, J., 2016. A civilian aircraft landing challenge. On-line available from the aerospace benchmark section of the SMAC Toolbox (http://w3. onera.fr/smac/).

Brockhaus, R., Alles, W., Luckner, R., 2013. Flugregelung. Springer, Berlin.

de Bruin, A., Jones, T., 2016. Accurate autonomous landing of a fixed-wing unmanned aircraft under crosswind conditions. In: IFAC-PapersOnLine. Vol. 49. pp. 170-175.

Doyle, J., Francis, B., Tannenbaum, A., 1990. Feedback Control Theory. Macmillan Publishing Co.

Doyle, J. C., Glover, K., Khargonekar, P. P., Francis, B. A., 1989. State-space solutions to standard $\mathrm{H}_{2}$ and $H_{\infty}$ control problems. IEEE Transactions on Automatic Control 34 (8), 831-847.

Francis, B. A., Wonham, W. M., 1975. The internal model principle for linear multivariable regulators. Applied Mathematics \& Optimization 2 (2), 170194.

Francis, B. A., Wonham, W. M., 1976. The internal model principle of control theory. Automatica 12 (5), 457-465.

Glover, K., Doyle, J. C., 1988. State-space formulae for all stabilizing controllers that satisfy an $H_{\infty}$-norm bound and relations to relations to risk sensitivity. Systems \& Control Letters 11 (3), 167-172.

Holley, W., Bryson, A., 1977. Wind modeling and lateral control for automatic landing. Journal of Spacecraft and Rockets 14 (2), 65-72.

Horowitz, I., 1963. Synthesis of Feedback Systems. Academic Press, New York.

Kaminer, I., Khargonekar, P. P., 1990. Design of the flare control law for longitudinal autopilot using $H_{\infty}$ synthesis. In: IEEE Conference on Decision and Control. pp. 2981-2986.

Lambregts, A., 1982. Avoiding the pitfalls in automatic landing control system 
design. In: AIAA Guidance and Control Conference.

Lambregts, A., Creedon, J., 1980. Development and flight evaluation of automatic flare laws with improved touchdown dispersion. In: AIAA Guidance and Control Conference. Paper No. 80-1757.

Looye, G., Joos, H.-D., 2006. Design of autoland controller functions with multiobjective optimization. Journal of Guidance, Control, and Dynamics 29 (2), 475-484.

Looye, G., Joos, H.-D., Willemsen, D., 2001. Application of an optimisationbased design process for robust autoland control laws. In: AIAA Guidance, Navigation, and Control Conference.

McRuer, D., Ashkenas, I., Graham, D., 1973. Aircraft Dynamics and Automatic Control. Princeton University Press, Princeton, NJ.

Navarro-Tapia, D., Simplicio, P., Iannelli, A., Marcos, A., 2017. Robust flare control design using structured $H_{\infty}$ synthesis: A civilian aircraft landing challenge. In: IFAC PapersOnLine. Vol. 50. pp. 2971-3976.

Shue, S.-P., Agarwal, R. K., 1999. Design of automatic landing systems using mixed $H_{2} / H_{\infty}$ control. Journal of Guidance, Control, and Dynamics 22 (1), $103-114$.

Skogestad, S., Postlethwaite, I., 2005. Multivariable Feedback Control. Prentice Hall, Upper Saddle River, NJ.

Stein, G., 2003. Respect the unstable. IEEE Control Systems Magazine, 12-25.

Theis, J., Ossmann, D., Pfifer, H., 2017. Robust autopilot design for crosswind landing. In: IFAC PapersOnLine. Vol. 50. pp. 3977-3982.

Zhou, K., Doyle, J., Glover, K., 1995. Robust and Optimal Control. Prentice Hall, Upper Saddle River, NJ. 